\title{
ASF1B promotes cervical cancer progression through stabilization of CDK9
}

\author{
Xinjian Liu' ${ }^{1,2}$, Jingwei Song ${ }^{1,3}$, Yenan Zhang ${ }^{1}$, Huiquan Wang ${ }^{1}$, Hongzhi Sun ${ }^{1}$, Xiaomin Feng ${ }^{4}$, Min Hou', Guo Chen ${ }^{5}$, \\ Qi Tang ${ }^{2}$ and Minjun Ji (iD)
}

\begin{abstract}
Cervical cancer (CC) is one of the most deadly cancers in women, its current treatments still result in poor outcomes and developing the novel targets and therapeutic strategies are urgently needed. Recent studies have shown that anti-silencing function 1B (ASF1B) might be used as a new proliferation marker for cancer diagnosis and prognosis. However, the expression and function of ASF1B in cervical cancer remain unclear. Here, we induced ASF1B knockdown and overexpression in cervical cancer cell lines and detected the biological behavior changes in vitro. Furthermore, we established two murine models using stable ASF1B-shRNA HeLa cells or normal HeLa cells following AAV-shRNAASF1B administration to evaluate how suppression of ASF1B affects tumor growth. We showed that ASF1B functions as an oncogene in cervical cancer cells. Silence of ASF1B suppressed cervical cancer cell growth in vitro and in vivo, while, ASF1B overexpression accelerated cancer cell proliferation. Furthermore, ASF1B deficiency induced cell cycle arrest and apoptosis. Mechanistically, we found that ASF1B formed stable complexes with cyclin-dependent kinase 9 (CDK9), and positively regulated CDK9 stabilization. Taken together, tumorigenic ASF1B could be targeted to suppress cervical cancer tumor growth by inducing apoptotic cell death.
\end{abstract}

\section{Introduction}

Cervical cancer is the fourth leading cause of cancer death in the world and the second most common female malignant tumor, with a mortality rate next only to only that of breast cancer ${ }^{1-3}$; there are $\sim 470,000$ new cases of $\mathrm{CC}$ each year, and the disease poses a great threat to the life of $\sim 276,000$ females ${ }^{4}$. Among these cases, approximately $90 \%$ occur in developing countries, and more than 50,000 females succumb to cervical cancer each year in China ${ }^{5,6}$. Our current understanding of cervical cancer is that persistent human papillomavirus (HPV) infection contributes to tumor establishment ${ }^{7-10}$. However, HPV infection alone is not sufficient for tumor development,

\footnotetext{
Correspondence: Minjun Ji (jiminjun@njmu.edu.cn)

${ }^{1}$ Department of Pathogen Biology, Nanjing Medical University, 211166 Nanjing, China

${ }^{2}$ Key Laboratory of Antibody Technique of National Health Commission of China, Nanjing Medical University, 211166 Nanjing, China

Full list of author information is available at the end of the article

These authors contributed equally: Xinjian Liu, Jingwei Song
}

Edited by S. Tait and host genetic factors may also be involved in cervical cancer pathogenesis ${ }^{11}$. Although clinical cervical cancer treatments, including surgery, chemotherapy, and radiotherapy, are usually performed, poor effects of clinical chemotherapeutic treatments have been observed ${ }^{12}$. Thus, the tumorigenesis mechanism of cervical cancer should be further studied, and new drug targets and treatment strategies should be sought.

As mentioned above, the molecular basis of cancer involves host genetic factors mutations ${ }^{13,14}$. Abnormal gene expression, including gene silencing or overexpression, is associated with variations in DNA methylation and aberrant histone post-translational modifications ${ }^{15-17}$. The deregulation of chromatin regulators has been shown to be involved in the occurrence and progression of cancer, including histone variant proteins, histone chaperone proteins, histone modifying enzymes, effector proteins and chromatin remodeling proteins ${ }^{18-20}$. Histone $\mathrm{H} 3-\mathrm{H} 4$ chaperone anti-silencing function 1 (ASF1) is an important histone chaperone protein that plays a role in the

\section{(c) The Author(s) 2020}

(c) (i) Open Access This article is licensed under a Creative Commons Attribution 4.0 International License, which permits use, sharing, adaptation, distribution and reproduction cc) in any medium or format, as long as you give appropriate credit to the original author(s) and the source, provide a link to the Creative Commons license, and indicate if changes were made. The images or other third party material in this article are included in the article's Creative Commons license, unless indicated otherwise in a credit line to the material. If material is not included in the article's Creative Commons license and your intended use is not permitted by statutory regulation or exceeds the permitted use, you will need to obtain permission directly from the copyright holder. To view a copy of this license, visit http://creativecommons.org/licenses/by/4.0/. 
chromatin-based progression of cellular DNA replication, DNA damage repair, and transcription regulation ${ }^{21,22}$. ASF1 exists as two paralogs: ASF1A and ASF1B. ASF1A contributes primarily to DNA repair and cell senescence, while ASF1B is preferentially involved in cell proliferation ${ }^{22,23}$. Interestingly, Armelle et al. revealed that a high mRNA level of ASF1B was correlated with clinical data and disease outcome in breast cancer, and the researchers proposed that ASF1B might be used as a new proliferation marker for breast cancer diagnosis and prognosis ${ }^{24}$.

Here, we induced ASF1B knockdown and overexpression in cervical cancer cell lines and detected the biological behavior changes in vitro. Furthermore, we established two murine models using stable ASF1BshRNA HeLa cells or normal HeLa cells following AAVshRNA-ASF1B administration to evaluate how suppression of ASF1B affects tumor growth. Our data implied that ASF1B was closely associated with proliferation, migration, and anti-apoptosis in cervical cancer cells and might be considered a novel therapeutic target and prognostic indicator in cervical cancer patients.

\section{Materials and methods}

\section{Patients and tissue samples}

We collected cervical cancer tumors and corresponding adjacent tissue samples from 50 patients who underwent tumor resection (The Affiliated Obstetrics and Gynecology Hospital of Nanjing Medical University). The protocol was approved by Nanjing Medical University Ethics Committee (No. 2019923). All tumors and matched nontumor specimens were diagnosed by pathology. No patients received chemotherapy and/or radiotherapy before surgery. The resected specimens were subjected to qPCR (12 samples) and to immunohistochemistry (IHC, 50 samples) using an anti-human ASF1B antibody (Invitrogen, US).

\section{Cell lines, cell culture, plasmid, reagents}

Human CaSki, HeLa, and HaCaT cells were obtained from Procell (Wuhan, China). The cell lines were maintained in Dulbecco's modified Eagle's medium (DMEM, Life) supplemented with $100 \mathrm{U} / \mathrm{ml}$ penicillin, $100 \mu \mathrm{g} / \mathrm{ml}$ streptomycin, $2 \mathrm{mmol} / \mathrm{l}$ L-glutamine, $10 \mathrm{mmol} / \mathrm{l} \mathrm{HEPES}$, and $10 \%$ fetal bovine serum (FBS) at $37^{\circ} \mathrm{C}$ in a $5 \% \mathrm{CO}_{2}$ humidified atmosphere. The cell lines have been confirmed as mycoplasma contamination free by Procell (Wuhan, China).

\section{Total RNA extraction and qPCR}

Total RNA was extracted using TRIzol reagent (Thermo Fisher, Shanghai, China) and reverse transcribed into cDNA using PrimeScript ${ }^{\mathrm{TM}}$ RT Master Mix (Takara, Dalian, China). qPCR was performed with a Roche
Detection System (Roche) in a $20-\mu$ l reaction mixture containing SYBR Green I. The expression data were normalized to the housekeeping gene GAPDH rRNA and were analyzed using the $2^{-\Delta \Delta C \mathrm{~T}}$ method.

\section{Knockdown and overexpression of ASF1B in stable cell lines}

ASF1B-shRNAs were designed, synthesized, and annealed to form double-strand DNA fragments that were cloned into the pGPU6/GFP/Neo vector (Gene Pharma, Shanghai, CN). The recombinant plasmid was transfected into human CaSki and HeLa cells with Lipofectamine ${ }^{\circledR 2} 2000$ (Invitrogen, US). The cells were incubated at $37^{\circ} \mathrm{C}$ in complete Opti-MEM medium containing $1200 \mu \mathrm{g} / \mathrm{ml}$ (HeLa) or $500 \mu \mathrm{g} / \mathrm{ml}$ (CaSki) G418 (Hanbio Biotechnology) for 30 days to select positive cells. A limited dilution strategy was performed to acquire the single cell clones. Total RNA from each clone was extracted, and qPCR was performed to explore whether the ASF1B gene was silenced. Protein expression levels were detected by western blot. The positive cells were selected and named ASF1B-shRNA cells.

The ASF1B overexpression plasmid pcDNA3.1-ASF1B was transfected into human CaSki and HeLa cells using Lipofectamine ${ }^{\circledR 2000 ~(I n v i t r o g e n) . ~ T h e ~ o v e r e x p r e s s i o n ~}$ level of ASF1B was identified by qPCR and western blot. Positive cells were selected and expanded to perform the following experiments.

\section{AAV-shRNA-ASF1B construction and AAV virus packaging}

The AAV-shRNA-ASF1B plasmid was constructed by Hanbio Biotechnology. Packaging of AAV-shRNA-ASF1B was performed as described before ${ }^{25}$. In brief, HEK 293 cells were transfected with a plasmid mix of pAAVshRNA-ASF1B, GV388, pH21, and pHELPER (molar ratio: 0.5 : $0.5: 1: 1)$ using Lipofectamine ${ }^{\circledR} 2000$. Twentyfour and forty-eight hours after transfection, the culture supernatants were collected and purified using Lenti- $\mathrm{X}^{\mathrm{TM}}$ Concentrator (Clontech, US). Viral titers were then determined using qPCR.

\section{Animal experiments}

Female nude mice (6 weeks old) were purchased from Beijing Vital River Laboratory Animal Technology Co., Ltd. All experiments with mice were performed according to a protocol approved by the Nanjing Medical University Animal Care and Use Committee (Approval number: IACUC-1705003). All mice received humane care according to the criteria outlined in the "Guide for the Care and Use of Laboratory Animals".

Model 1: Mice were divided randomly into two groups ( $n=5$ /group): the ASF1B-shRNA group and the scrambled shRNA control group. Next, $5 \times 10^{6}$ stable ASF1B-shRNA HeLa cells or corresponding scrambled 
cells with 15\% Matrigel (BD, \#354248) in $100 \mu$ l PBS were injected subcutaneously into the flank of each nude mouse. Tumor sizes were measured by calipers every 3 days. The tumor volumes were calculated as follows: tumor volume $\left(\mathrm{mm}^{3}\right)=1 / 2 \times$ length $\times$ width $^{2}$.

Model 2: Subcutaneous tumors following AAV-shRNAASF1B administration were also investigated. In brief, nude mice were also divided randomly into two groups ( $n=5$ /group): the AAV-shRNA-ASF1B group and the scrambled AAV control group. Next, $5 \times 10^{6} \mathrm{HeLa}$ cells with $15 \%$ Matrigel in $100 \mu \mathrm{l}$ PBS were injected subcutaneously into the flank of each nude mouse. AAVshRNA-ASF1B or scrambled AAV-shRNA at a dose of $5 \times 10^{11}$ viral particles was injected directly into the tumors on days $12,15,18$, and 27 . Tumor sizes were measured every 3 days. Seven weeks later, all mice were sacrificed, and the tumors were excised, harvested, weighed, and fixed in formalin for at least $24 \mathrm{~h}^{26-28}$.

\section{Western blot analysis}

To lyse cells, cell pellets were resuspended in cold lysis protein extraction reagent (Thermo Fisher Scientific, Inc) with a protease inhibitor cocktail (Sigma); the protein was quantified and then used to perform western blot as previously described $^{29}$. Antibodies against ASF1B, cyclin A1, cyclin B1, cyclin D1, cleaved caspase 3, Bcl-2, Bax, and $\beta$-actin were purchased from Cell Signaling (Danvers, MA). Mouse anti-CDK9 antibody was obtained from Novus (Centennial, CO) and rabbit anti-CDK9 were purchased from Cell signaling (Danvers, MA).

\section{Cell viability assay}

Cell viability analysis was performed using a Cell Counting Kit-8 (CCK-8; Beyotime Institute of Biotechnology, Shanghai, China). Briefly, $2 \times 10^{3}$ cells were seeded into 96-well plates. At $24 \mathrm{~h}, 48 \mathrm{~h}$ and $72 \mathrm{~h}, 10 \mu \mathrm{l}$ CCK- 8 solution was added to each well, and the cells were incubated for $3 \mathrm{~h}$ at $37^{\circ} \mathrm{C}$. Then, the absorbance was measured using a microplate reader (Bio-tech, CA, USA) with a $450 \mathrm{~nm}$ wavelength.

\section{Colony formation assay}

Cells grown to $90 \%$ confluence were harvested and seeded in $10-\mathrm{cm}$ plates at 300 cells per plate. Fourteen days later, after washing with PBS, the cells were stained with a $0.05 \%$ crystal violet solution for imaging and colony counting.

\section{Wound healing assay}

Cells were seeded in 6-well plates until their growth confluence reached $90 \%$. Next, 2-mm scratches were performed using a $200-\mu \mathrm{l}$ pipette tip. The cell-free gaps were measured over time with an optical microscope (Zeiss).

\section{Invasion assay}

A total of $50 \mu \mathrm{g} / \mathrm{cm}^{2}$ reconstituted Matrigel (SigmaAldrich) was used to coat polycarbonate filters $(8 \mu \mathrm{m}$; Corning, NY, USA). Next, $2 \times 10^{3}$ cells were seeded into the upper chamber with $200 \mu \mathrm{l}$ serum-free DMEM. DMEM with $10 \%$ FBS was added to the lower chamber, and the cells were cultured at $37^{\circ} \mathrm{C}$ in a $5 \% \mathrm{CO}_{2}$ humidified atmosphere. Twenty-four and forty-eight hours later, non-invading cells were removed, and the cells on the lower surface were counted microscopically ${ }^{30}$.

\section{Flow cytometry for analysis of the cell cycle and apoptosis}

To perform cell cycle assays, cells were fixed with 70\% $\mathrm{EtOH}$ for $15 \mathrm{~min}$ and then washed with PBS. Cell pellets were suspended in $500 \mu \mathrm{l}$ PI solution (BD Biosciences, San Jose, CA) and incubated for $40 \mathrm{~min}$ at $37^{\circ} \mathrm{C}$. Before washing, $3 \mathrm{ml}$ PBS was added. The cells were pelleted, and the supernatant was removed; then, the pellet was suspended in $500 \mu \mathrm{l}$ PBS for flow cytometry analysis.

Cell apoptosis analysis was performed with a PE Annexin V Apoptosis Detection Kit (Beyotime, Shanghai, China) according to the manufacturer's protocol. In brief, after washing with cold PBS twice, the cells were resuspended in binding buffer. A total of $1 \times 10^{5}$ cells $(100 \mu \mathrm{l})$ were transferred to the flow tube, and $5 \mu \mathrm{l}$ of PE Annexin $\mathrm{V}$ and $5 \mu \mathrm{l} 7$-AAD were added. After gentle vortexing and incubation for $15 \mathrm{~min}$ at room temperature in the dark, another $400 \mu \mathrm{l}$ binding buffer was added and then analyzed by flow cytometry.

\section{Immunohistochemistry}

Tumor tissue sections were harvested and treated as previously described ${ }^{31}$. Primary antibodies for anti-human ASF1B (1:100), anti-mouse ASF1B, Ki67, and CD31 (1:100) were purchased from Cell Signaling Technologies. An ImmPRESS ${ }^{\mathrm{TM}}$ HRP Anti-Rabbit IgG (Peroxidase) Polymer Detection Kit, raised in horse (Vector Laboratories), was used for the secondary antibody. ImmPACT DAB Peroxidase (HRP) Substrate was used for detection, and hematoxylin (Vector Laboratories) was used as a counterstain.

\section{Protein-protein interaction studies}

Co-IP was conducted as previously described ${ }^{32}$. Briefly, whole-cell extracts of Flag-ASF1B-transfected HeLa cells were prepared, and immunoprecipitation was performed by adding $20 \mu \mathrm{l}$ of anti-Flag affinity sepharose or normal iso-type antibody and incubating overnight at $4{ }^{\circ} \mathrm{C}$. Finally, the immune complex was detected by Western blot. As controls, samples without treatment were used. Then, the Co-IP product was separated by SDS-PAGE. The whole gel was subjected to silver staining and then liquid chromatography-mass spectrometry (LC-MS) analysis $^{33}$. 
The LC-MS analysis results indicated that cyclindependent kinase 9 (CDK9) might be involved in the progression of cervical tumors. To further confirm this hypothesis, Co-IP was performed using an anti-ASF1B antibody or anti-CDK9 antibody. Antibodies against ASF1B and CDK9 for Co-IP were purchased from Novus Biologicals (Colorado, US). To test the protein stability, cells were incubated with $20 \mu \mathrm{g} / \mathrm{ml}$ Cycloheximide (CHX, SigmaAldrich) for the indicated periods of time. To further determine whether ASF1B promote the proteasomal stabilization of CDK9, ASF1B-shRNA HeLa cells or control cells were treated with or without proteasome inhibitor MG132. The treated cell proteins were extracted for western blot.

\section{Cell immunofluorescence staining}

For double immunofluorescence staining, cells grown on coverslips were prepared and fixed in $4 \%$ paraformaldehyde for $15 \mathrm{~min}$. Then, the cells were treated with $0.3 \%$ Triton X-100 for 20 min and blocked with $10 \%$ normal goat serum for an hour. The cells were then incubated with primary antibodies (1:100), including rabbit anti-human ASF1B mAb (Cell Signaling, Danvers, $\mathrm{MA}$ ) and mouse anti-human CDK9 mAb (Novus Biologicals, Colorado, USA) at $4{ }^{\circ} \mathrm{C}$ overnight. The cells were washed with PBS and then incubated with the appropriate Alexa-488- or TRITC-conjugated secondary antibodies (1:200; Proteintech, Chicago, USA) at room temperature for $60 \mathrm{~min}$. The cells were counterstained with DAPI and photographed using a Zeiss Axio Imager and microscope LSM710 (Carl Zeiss, Jena, Germany).

\section{Statistics analysis}

Paired data were analyzed using a 2-tailed paired Student's $t$ test. A $p$ value of $<0.05$ was considered significant.

\section{Results}

\section{Patients' characteristics}

Primary characteristics of cervical cancer patients were shown in Table 1. The median age of patients was 53.5 years, the age range was from 26 to 73 years old and $82 \%$ $(41 / 50)$ were over 35 years. Histopathological results revealed that $98 \%(49 / 50)$ of cases were of cervical squamous cell carcinoma, and $2 \%(1 / 50)$ were of adenocarcinoma. In line with the FIGO staging, the clinical staging was carried out: 27 cases were stage I and 18 cases were stage II. According to the WHO classification, the pathological grades were classified into groups with 2 cases (4\%) highly differentiated carcinoma, 39 cases (78\%) moderately differentiated, and 9 cases (18\%) poorly differentiated.

\section{ASF1B expression was upregulated in cervical cancer tissues and stable cell lines}

ASF1B has been shown to promote breast cancer, prostate cancer, cell renal cell carcinoma ${ }^{24,34,35}$. However,
Table 1 Association between ASF1B expression and clinicopathologic parameters of cervical cancer patients.

\begin{tabular}{|c|c|c|c|c|c|c|c|}
\hline \multirow{3}{*}{$\begin{array}{l}\text { Characteristic } \\
\text { Age, years }\end{array}$} & \multirow{3}{*}{$\frac{\text { Total }}{50}$} & \multicolumn{4}{|c|}{ ASF1B expression } & \multirow{3}{*}{ Statistics } & \multirow{3}{*}{$P$} \\
\hline & & \multicolumn{2}{|c|}{ low } & \multicolumn{2}{|c|}{ high } & & \\
\hline & & & Mean & & Mean & & \\
\hline$\leq 35$ & 5 & 3 & 48.24 & 2 & 52.12 & 1.204 & 0.2344 \\
\hline$>35$ & 45 & 30 & & 15 & & & \\
\hline FIGO stage & 45 & & & & & & \\
\hline 1 & 27 & 21 & & 6 & & 0.6818 & 0.4090 \\
\hline$\|$ & 18 & 12 & & 6 & & & \\
\hline Tumor size $(\mathrm{cm})$ & 50 & & & & & & \\
\hline$<4$ & 44 & 30 & 1.700 & 14 & 3.029 & 2.652 & $0.0108^{*}$ \\
\hline$\geq 4$ & 6 & 3 & & 3 & & & \\
\hline $\begin{array}{l}\text { Deep stromal } \\
\text { invasion } \triangle\end{array}$ & 34 & & & & & & \\
\hline$<2 / 3$ & 9 & 7 & & 2 & & & 0.6921 \\
\hline$\geq 2 / 3$ & 25 & 17 & & 8 & & & \\
\hline $\begin{array}{l}\text { Lymphovascular } \\
\text { space invasion } \triangle\end{array}$ & 25 & & & & & & \\
\hline Negative & 18 & 10 & & 8 & & & 0.0573 \\
\hline Positive & 7 & 7 & & 0 & & & \\
\hline Nerve invasion & 49 & & & & & & \\
\hline Negative & 41 & 27 & & 14 & & 0.2546 & 0.6138 \\
\hline Positive & 8 & 6 & & 2 & & & \\
\hline
\end{tabular}

NOTE: These four clinico pathologic parameters, including FIGO stage, Deep stromal invasion, Lymphovascular space invasion and nerve invasion, have some missing samples. $\Delta$ : Fisher's exact test $(n<40)$

its expression level and role in cervical cancer remain to be elucidated. To investigate the role of ASF1B in cervical cancer tumor progression, the expression level of ASF1B was assessed with paired cervical tumors and adjacent tissues from 12 patients by qPCR. Compared with that in adjacent tissues, ASF1B was remarkably increased in tumor tissues $(p<0.05, n=12)$ (Fig. 1a). To further evaluate the level of ASF1B, cervical cancer tissue and corresponding adjacent tissue samples from 50 patients were tested by immunohistochemistry. As shown in Fig. 1b, c, positive staining for ASF1B was stronger in cervical cancer tissues than in adjacent tissues $(p<0.05)$. We also explored the correlation between ASF1B level and clinical cervical tumor patients' features ${ }^{36}$. Upregulation of ASF1B was positively correlated with tumor size $(p=0.0108$, Table 1$)$. These data indicated the possible involvement of ASF1B cervical cancer cells proliferation.

Next, we examined whether ASF1B was overexpressed in different cervical cancer cell lines: CaSki and HeLa 


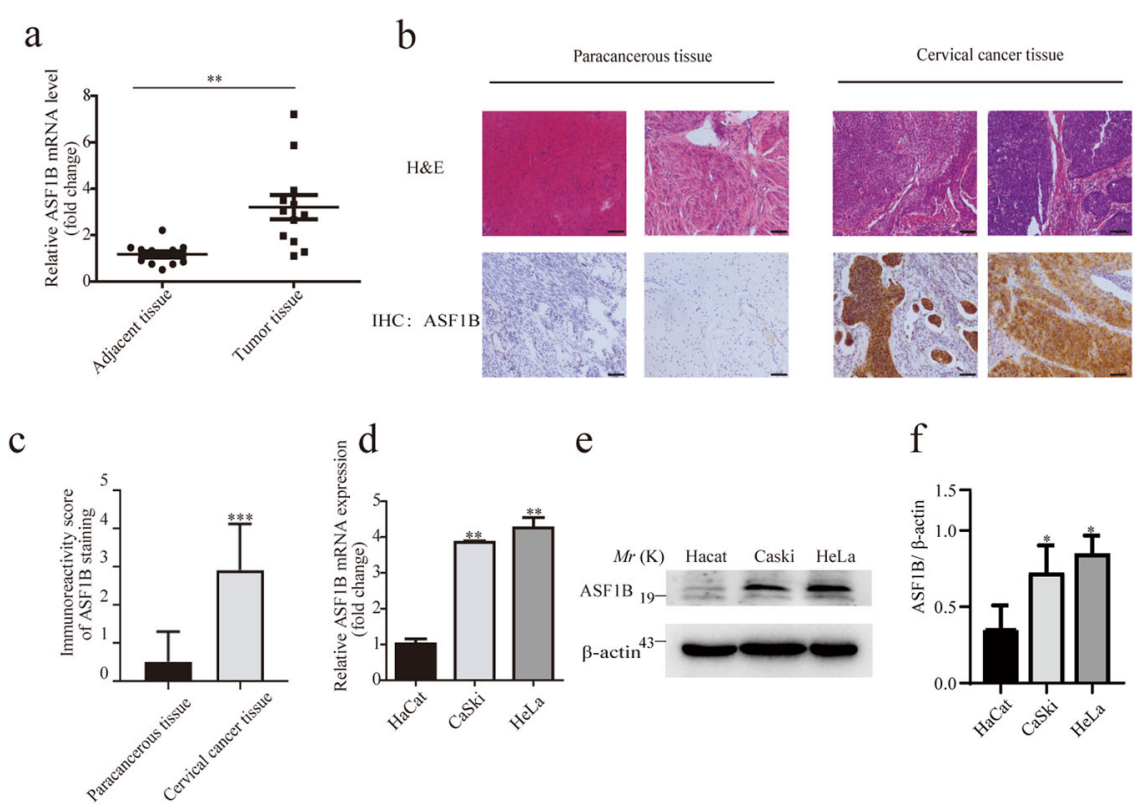

Fig. 1 ASF1B expression in cervical tumors, adjacent tissues and human cervical cancer cell lines. a The expression level of ASF1B was assessed in paired cervical cancer and adjacent tissues from 12 patients by qPCR. $\mathbf{b}$ Pairs of cervical cancer tissue and paracancerous tissue samples from 50 patients were tested by H\&E and immunohistochemistry. c Immunoreactivity score of ASF1B staining from the immunohistochemistry results. $\mathbf{d}$ Levels of ASF1B mRNA expression in cervical cancer cell lines (CaSki and HeLa) and a control cell line (HaCat). e The protein expression of ASF1B in CaSki, HeLa and HaCat cells by western blot. A higher level of ASF1B was detected in CaSki and HeLa cells than that in HaCat cells. $\mathbf{f}$ Quantification of the results in (e). Data are mean \pm SEM, $n=3$, and two-tailed unpaired Student's $t$ test was used. ${ }^{* * *} p<0.001$.

cells. As shown in Fig. 1d, the qPCR analysis results showed that ASF1B mRNA levels were higher in CaSki cells and HeLa cells than in $\mathrm{HaCaT}$ cells, which is a spontaneously transformed aneuploid immortal keratinocyte cell line from adult human $\operatorname{skin}^{37}$. Western blot results further indicated that the level of ASF1B protein expression was much higher in CaSki cells and HeLa cells than in $\mathrm{HaCaT}$ cells (Fig. 1e, f). All these data illustrated the differential expression of ASF1B in distinct cervical cancer cells.

Knockdown or overexpression of ASF1B was mediated by shRNA or recombinant plasmid in cervical cancer cell lines

To study the role of ASF1B in cervical cancer, shRNA technology was used to knock down ASF1B. Oligonucleotides were synthesized and then annealed to make a double-stranded DNA fragment that was inserted into the pGPU6/GFP/Neo vector. This recombinant vector or scrambled vector was transfected into CaSki cells and HeLa cells with Lipofectamine ${ }^{\circledR} 2000$ (Invitrogen). With limited dilution strategies, a single cell clone was selected (Fig. 2a), and the mRNA and protein levels were detected by qPCR and western blot. The results from western blot (Fig. 2b, c) validated that the ASF1B level was reduced and that ASF1B knockdown was successful in CaSki cells and HeLa cells; thus, the corresponding stable ASF1B knockdown cell lines were established.
Ectopic expression was also induced by plasmidmediated technology. pcDNA3.1-ASF1B was transfected into human CaSki and HeLa cells (Fig. 2d). Increased ASF1B mRNA and protein expression levels were detected by western blot (Fig. 2e, f).

\section{Knockdown of ASF1B inhibited cervical cancer cell proliferation}

A CCK-8 assay was conducted to examine cancer cell proliferation. The results showed that ASF1B knockdown led to a significant reduction in cell viability (Fig. S1a, S1b). A colony formation assay was also used to investigate the effect of ASF1B on cervical cancer cell growth. The images showing colony formation and clone number demonstrated that ASF1B knockdown significantly suppressed HeLa cells and CaSki cells $(p<0.01$, Fig. S1c, S1d). In contrast, ectopic expression of ASF1B significantly accelerated cancer cell proliferation as evidenced by CCK-8 assay (Fig. S1e, S1f) and induced cell growth according to colony formation assay (Fig. S1g, S1h). These results suggested that ASF1B alteration significantly inhibits cervical cancer cell proliferation and anchorage-free colony formation.

\section{Knockdown of ASF1B suppressed cervical cancer cell migration}

Wound healing assays were performed to test whether the ASF1B gene impacts cervical cancer cell motility. 
a

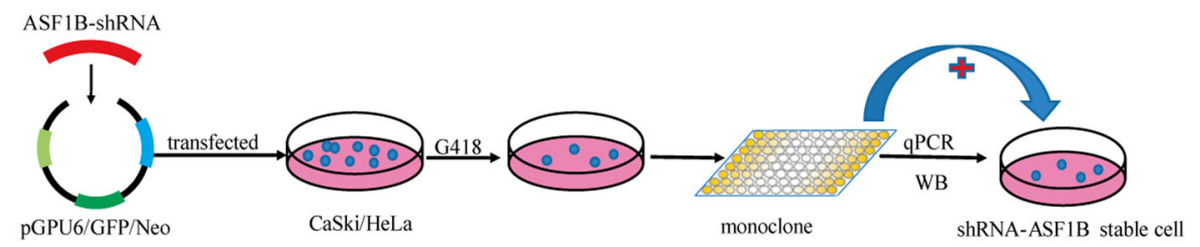

$\mathrm{b}$

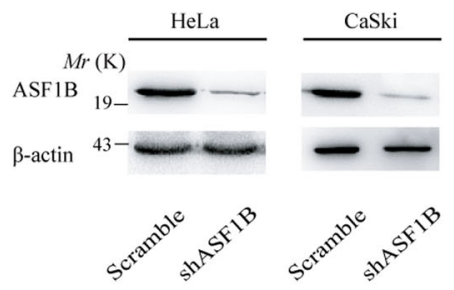

$\mathrm{d}$

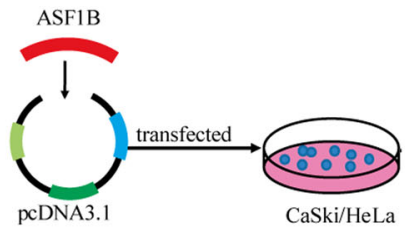

e

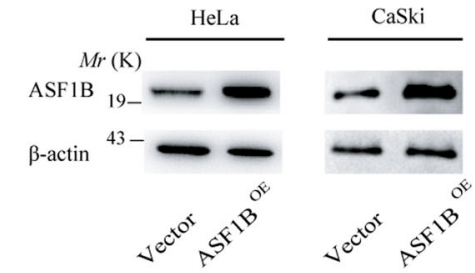

$\mathrm{c}$
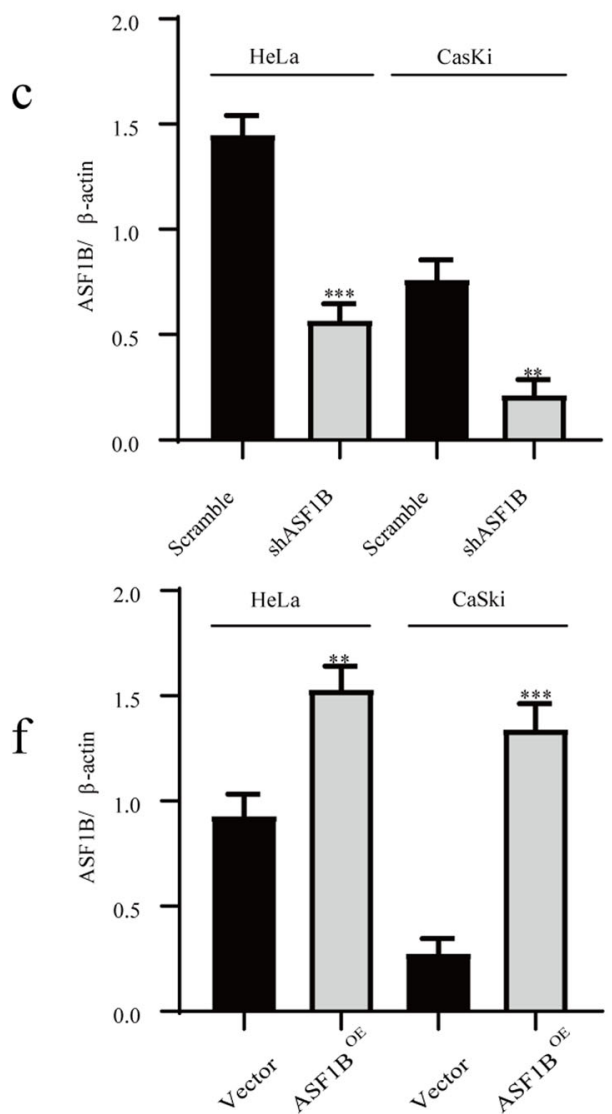

Fig. 2 Knockdown or overexpression of ASF1B in cervical cancer cells. a A total of $1 \times 10^{5}$ CaSki or HeLa cells were seeded in each well of a 6-well plate, and the recombinant plasmid ASF1B-shRNA was transfected into cells with Lipofectamine ${ }^{\circledR} 2000$. The cells were incubated in complete Opti-MEM medium containing G418 for 30 days to select positive cells. A limited dilution strategy was performed, and the positive cell clones were selected. b ASF1B protein expression in stable ASF1B-shRNA-cells. Western blot detecting lower ASF1B protein levels in stable ASF1B-shRNA-CaSki or ASF1B-shRNA-HeLa cells than those in control cells. c Quantification of the results in (b). Data are mean $\pm \mathrm{SEM}, n=3$, and two-tailed unpaired Student's $t$ test was used. ${ }^{* *} p<0.001$. d. Establishment of ASF1B overexpression in CaSki or HeLa cells with overexpression technology. pcDNA3. 1-ASF1B was transfected into human CaSki and HeLa cells using Lipofectamine ${ }^{\circledR 2000}$. e Western blot results showed that observable ASF1B protein was detected in ASF1B-CaSki and HeLa cells relative to that in control cells. $\mathbf{f}$ Quantification of the results in (e). Data are mean $\pm S E M, n=3$, and twotailed unpaired Student's $t$ test was used. ${ }^{* *} p<0.001$.

Representative images and the quantified results indicated that ASF1B knockdown significantly suppressed cancer cell motility (Fig. S2a, S2b). To further examine the effect of ASF1B on cell invasion, a transwell assay was also performed. Compared with the scrambled cervical cancer cells, ASF1B-shRNA-transfected cervical cancer cells showed significantly decreased invasiveness $(p<0.01$, Fig. S2c, S2d). Wound healing and transwell assay results showed that knockdown of ASF1B significantly inhibited migration and invasion. Conversely, ectopic expression of ASF1B accelerated cancer cell migration $(p<0.01$, Fig. S2e, S2f) and increased invasiveness in cervical cancer cells $(p<0.01$, Fig. S2g, S2h).

\section{Knockdown of ASF1B in cervical cancer cells slowed tumor growth in the recipient mice}

ASF1B downregulation inhibited cell growth and migration in cervical cancer cells in vitro, which 
a

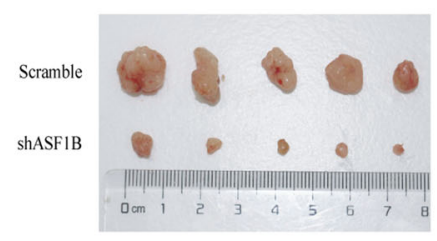

c

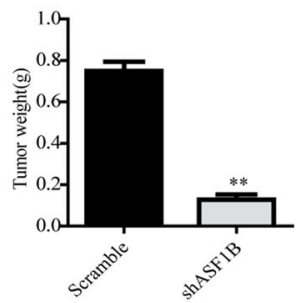

e

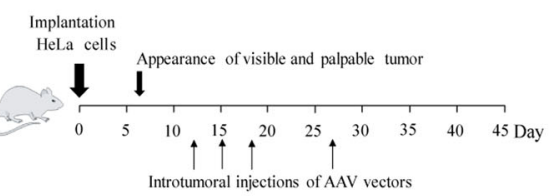

g

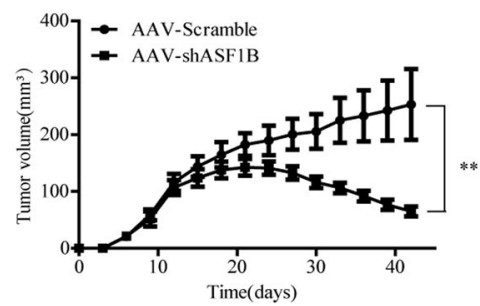

b

d

f

$\mathrm{h}$
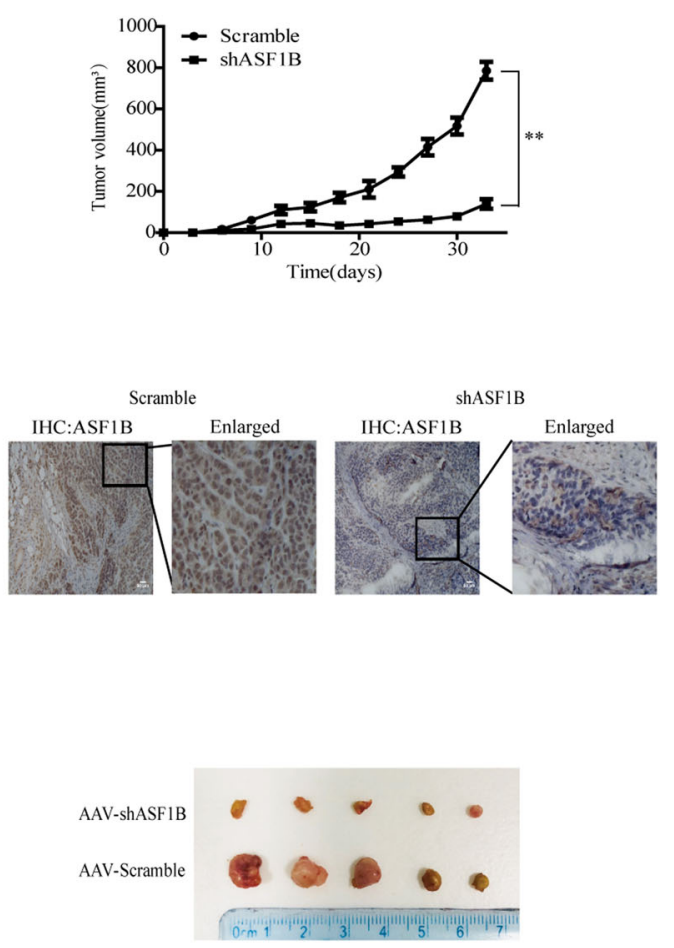

Fig. 3 Knockdown of ASF1B in HeLa cells or xenograft tumors in vivo following AAV-shRNA-ASF1B administration suppressed tumor growth. Stable ASF1B-shRNA-HeLa cells, as well as their relative controls, were prepared and harvested. A total of $5 \times 10^{6}$ cells were injected subcutaneously into the flank of each nude mouse. Tumor sizes were measured every three days. a Images of tumors after each mouse was euthanized. b Measured tumor volumes. The data show that the ASF1B-shRNA tumors grew more slowly than the scrambled control tumors. c Accumulated tumor weights are shown for mice receiving ASF1B-shRNA HeLa cells and control cells. $\mathbf{d}$ The tumors were harvested and then fixed with formalin to prepare slides. IHC was performed to detect the expression of ASF1B. e A total of $5 \times 10^{6}$ HeLa cells were injected subcutaneously into the flank of each nude mouse. AAV-shRNA-ASF1B or AAV-scrambled was injected into the formed tumors, and growth was observed on days 12 , 15, 18, and 27. f Images of tumors after each mouse was euthanized. g Measured tumor volumes. The mean volume of tumors after administration was significantly smaller for the AAV-shRNA-ASF1B group than for the scrambled AAV group. $\mathbf{h}$ The tumors were harvested and then fixed with formalin to prepare slides. IHC was performed to detect the expression of ASF1B.

encouraged us to investigate the effects of ASF1B on tumor growth in vivo. To this end, $5 \times 10^{6}$ ASF1BshRNA-HeLa or scrambled control cells were injected subcutaneously into the flank of each nude mice. Tumor sizes were measured every three days. The measured data showed that the tumors grew more slowly than those of the scrambled control, and this difference increased until the endpoint of 7 weeks (Fig. 3a, b). Consistently, ASF1B-shRNA-HeLa tumors were smaller in size and lower in weight compared to scrambled control tumors (Fig. 3c). IHC results indicated that ASF1B expression was decreased in ASF1BshRNA tumors compared to that in control tumors (Fig. 3d). 
Xenograft tumors were reduced in vivo following AAV-shRNA-ASF1B administration

To further explore the effect of ASF1B silencing in vivo, AAV-shRNA-ASF1B was constructed and packed into an AAV virus. Viral titers were determined using qPCR. HeLa cells were harvested and injected subcutaneously into the flank of each nude mouse at a dose of $5 \times 10^{6}$ cells per mouse, and tumor formation was observed. AAVshRNA-ASF1B or AAV-scrambled at a dose of $5 \times 10^{11}$ viral particles was injected into the formed tumors at days 12, 15, 18, and 27 (Fig. 3e). The mean volume of HeLaderived tumors after administration was significantly smaller for the AAV-shRNA-ASF1B treated group $(n=5)$ than for the scrambled AAV group $(n=5)(p<0.01$, Fig. 3f, g). Interestingly, the average tumor size reached its peak $\left(141.31 \mathrm{~mm}^{3}\right) 12$ days after the administration of AAV-shRNA-ASF1B, and the tumor size then decreased gradually. Finally, the mean tumor size in the AAV-shRNA-ASF1B-treated group was only $65.43 \mathrm{~mm}^{3}$, while the average size was $253.2 \mathrm{~mm}^{3}$ in the control group at their life endpoint. These data further supported the notion that ASF1B silencing led to the inhibition of tumor growth in cervical cancer cells. IHC results also demonstrated the decreased expression of ASF1B in tumors with AAV-shRNA-ASF1B administration (Fig. 3h).

\section{ASF1B knockdown suppressed HeLa cell proliferation by arresting the cell cycle and regulating the apoptotic pathway}

To explore the molecular mechanism of the ASF1B knockdown-mediated suppression of cervical cancer cell growth, the cell cycle was also investigated. We found that ASF1B inhibition caused G2 phase cell cycle arrest and significantly inhibited the cell cycle (Fig. 4a, b). Similarly, a panel of well-characterized signaling molecules of the cell cycle was detected in ASF1B-shRNA cells and control cells by western blot. As shown in Fig. 4c, weaker bands for the cyclin A1, cyclin B1 and cyclin D1 proteins were shown in ASF1B-shRNA-HeLa cells compared to those in control cells. These results indicated that knockdown of ASF1B induced cell cycle arrest, which mediated the inhibition of cervical cancer cell growth. We also monitored the cell cycle and the signaling molecules of the cell cycle in ASF1B-expressing HeLa cells. Interestingly, there was no significant change in the cell cycle (Fig. 4d, e), although the bands for the cyclin A1, cyclin B1 and cyclin D1 proteins were increased (Fig. 4f).

Next, cell apoptosis was also measured to elucidate the molecular mechanism of ASF1B knockdown. The results showed that more cells were apoptotic in ASF1B-shRNA HeLa cells than in scrambled cells (Fig. 5a, b). Next, a panel of apoptotic signaling molecules was examined in ASF1B-shRNA cells and control cells by western blot. As shown in Fig. 5c, d, stronger bands for Bax and cleaved caspase 3 and a weaker band for $\mathrm{Bcl}-2$ were detected in ASF1B-shRNA-HeLa cells compared to those in control cells, suggesting that knockdown of ASF1B induced apoptotic cell death.

Ki67 is a well-known proliferation marker for evaluating cell proliferation ${ }^{38}$, and CD31 is a useful marker for evaluating tumor microvessel density ${ }^{39}$. Thus, we also performed IHC staining for Ki67 and CD31. IHC staining demonstrated decreased Ki67 and CD31 levels in tumors with AAV-shRNA-ASF1B administration compared to those in tumors with AAV-scrambled treatment (Fig. 5e).

\section{CDK9 was involved in the progression of ASF1B-mediated} HeLa cell proliferation

To further explore the mechanism of ASF1B, LC-MS was performed to screen the interaction proteins of ASF1B. Ectopic ASF1B-expressing HeLa cells were extracted for IP, and western blot was conducted to verify IP efficiency (Fig. 6a). IP products were subjected to SDSPAGE electrophoresis (Fig. 6b) and LC-MS. LC-MS results showed that some proteins could bind to ASF1B, including MD2H, FOXF1, and CDK9 (Table 2). Interestingly, we found that $\mathrm{CDK} 9$, which is required for gene silencing ${ }^{40}$ and plays an important role in the pathogenesis $^{41,42}$ in cancer cells, was involved, and we speculated that CDK9 might be an important transcription factor for ASF1B to mediate the occurrence and progression of cervical cancer. We subjected the ASF1B and CDK9 genes to the website (http://genemania.org/) to predict the relationship between these two proteins. The results indicated that ASF1B could bind CDK9 via ASF1BMSH2-CDK9, ASF1B-KHSRP-CDK9 or ASF1B-HIRACDK9 (Fig. S3). Following, we detected the mRNA and protein levels of CDK9 in stable knockdown ASF1B cells and scrambled cells. To our surprise, ASF1B knockdown reduced CDK9 protein expression, but the mRNA level of CDK9 was not altered in stable ASF1B-shRNA cells (Fig. 6c, d). Co-IP was then performed with anti-ASF1B or anti-CDK9 antibodies using normal HeLa cells. Figure 6e shows that endogenous ASF1B formed stable complexes with CDK9.

To further elucidate the underlying mechanism of ASF1B and CDK9 in cervical cancer progression, we hypothesized that ASF1B knockdown reduces CDK9 protein levels by promoting its degradation. $\mathrm{CHX}$, a de novo protein biosynthesis inhibitor, was used to treat stable ASF1B knockdown cells or scrambled cells. We found that compared with the vector control, ASF1B knockdown reduced the stability of CDK9 protein (Fig. 6f). Treatment with MG132 induced to an increase in CDK9 levels in ASF1B-shRNA HeLa cells compared to control cells (Fig. 6g, h). Together, these data demonstrated that ASF1B promote proteasomal stabilization of CDK9.Then, immunofluorescent staining and imaging 


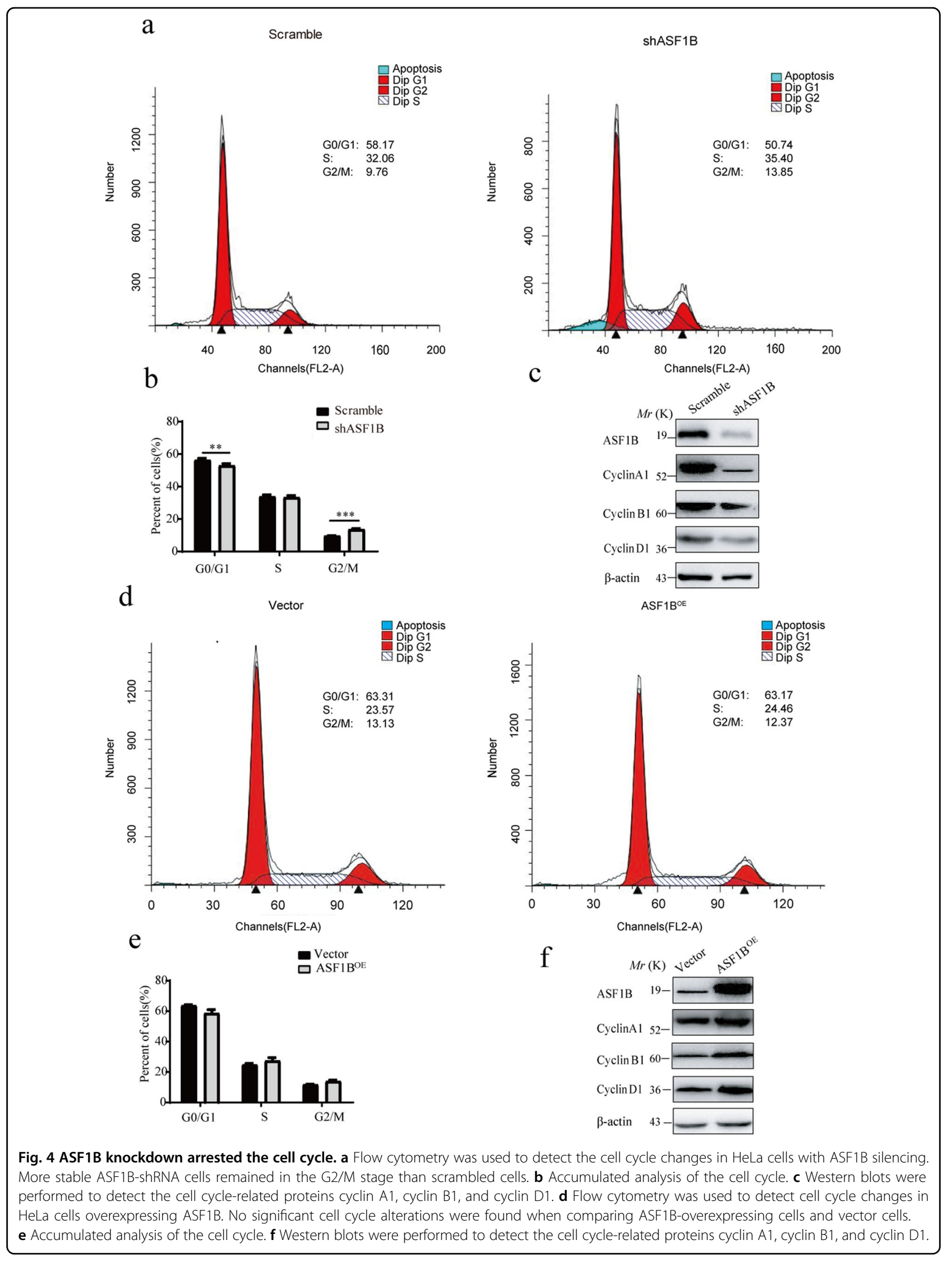



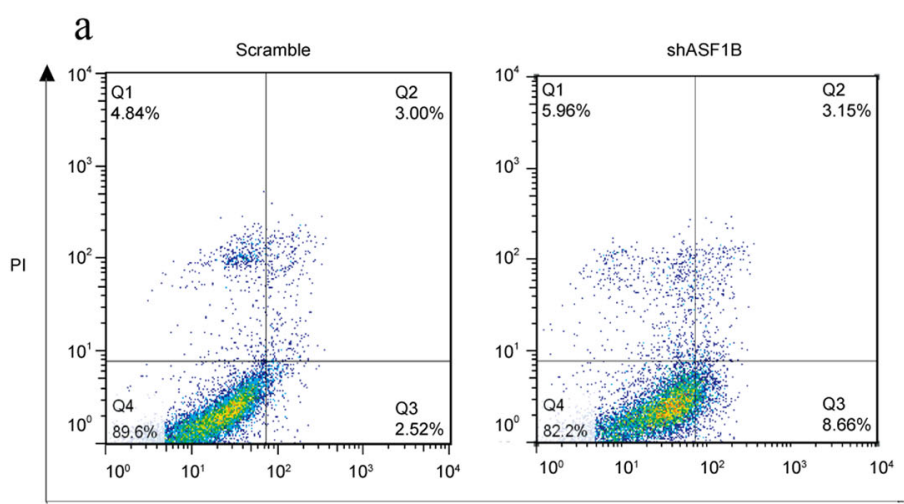

b

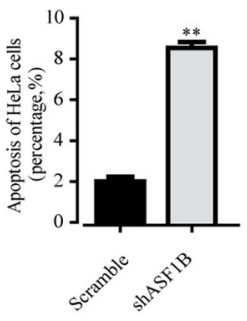

AnnexinV-FITC

$\mathrm{c}$
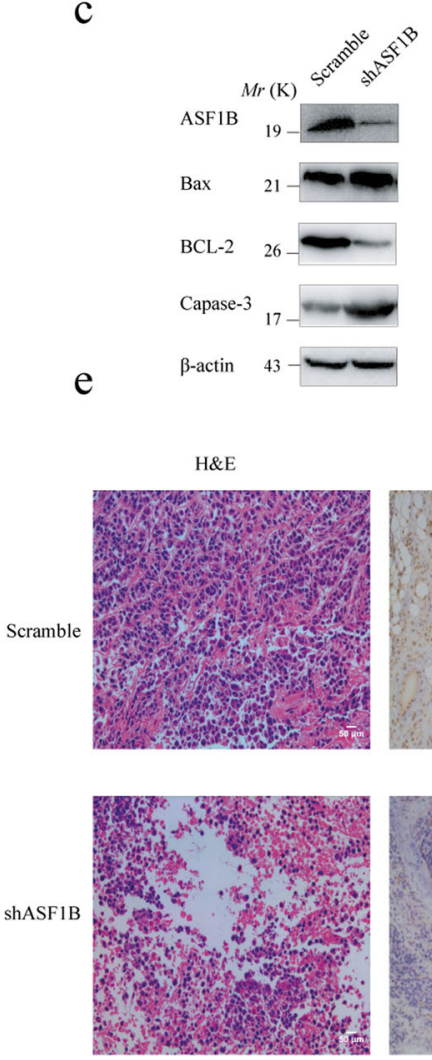

d

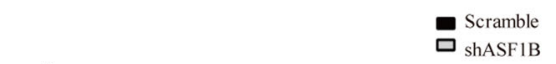

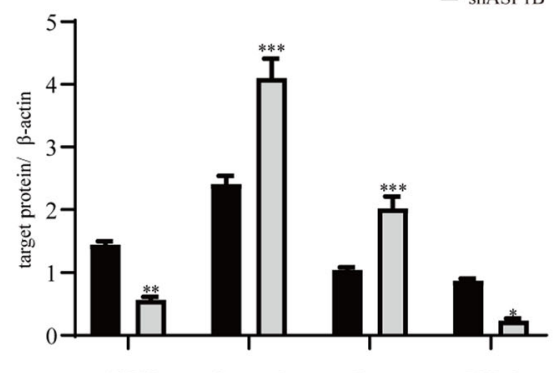

ASF1B

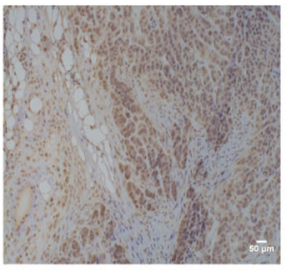

$\mathrm{CD} 31$
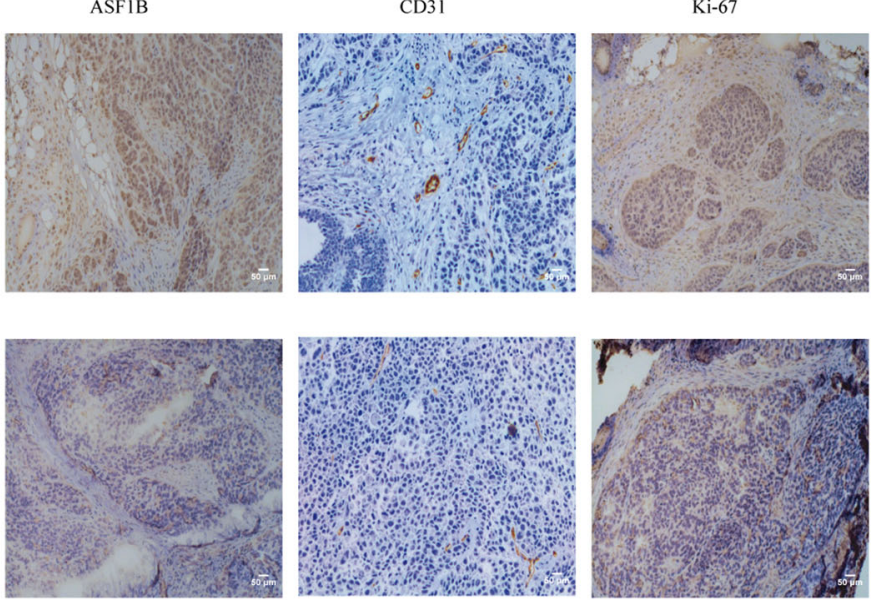

Fig. 5 ASF1B knockdown induced apoptotic HeLa cell death by suppressing the Bcl-2/ Bax /caspase apoptotic pathways. a, b Flow cytometry was used to detect the apoptosis of HeLa cells with ASF1B silencing. Stable ASF1B-shRNA-HeLa cells and control cells were seeded into 6-well plates. After washing and staining with PE Annexin $V$ and 7-AAD, flow cytometry analysis was performed. c Protein expression of canonical molecules important for cell apoptosis. Western blot was used to detect the protein levels of Bax, Bcl-2, and cleaved caspase 3 in the indicated cells. d Quantification of the results in (c). Data are mean \pm SEM, $n=3$, and two-tailed unpaired Student's $t$ test was used. ${ }^{* *} p<0.001$. e H\&E staining shows the tumor structure; $I H C$ was performed to detect the expression of ASF1B, CD31, and Ki67.

were used to visualize the colocalization of ASF1B and CDK9 in stable ASF1B-shRNA HeLa cells and corresponding scrambled cells. The co-staining images of ASF1B (green fluorescence) and CDK9 (red fluorescence) indicated that ASF1B was present in the nucleus and co-localized with CDK9 in scrambled cells, and the immunofluorescent signal of CDK9 was also weak in the nucleus following ASF1B knockdown (Fig. 6i) ${ }^{43}$.

Taken together, these results suggest that impaired expression of ASF1B inhibits cervical cancer growth and induces apoptosis, which is associated with modulation by the ASF1B/CDK9 pathways (Fig. 6j). 


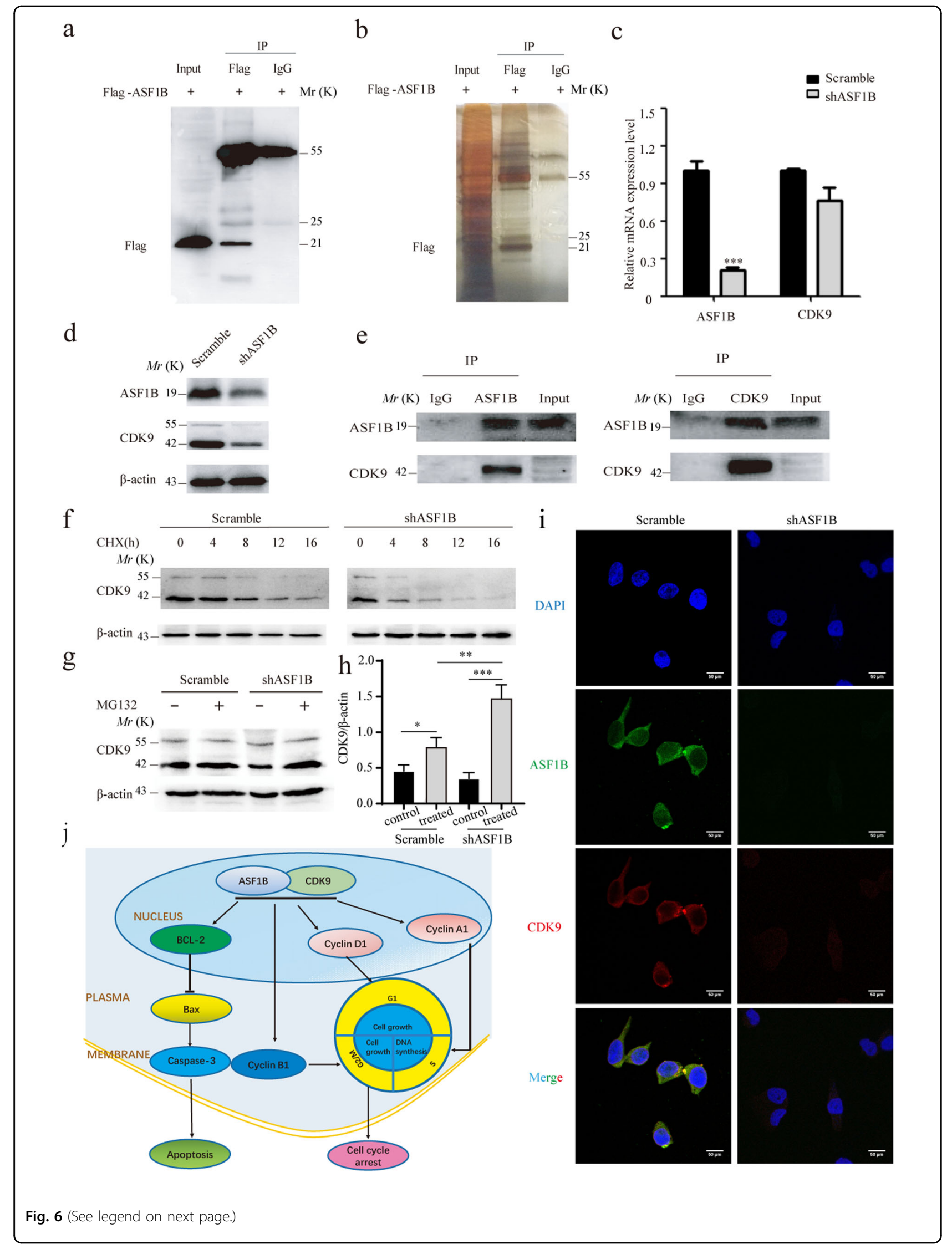


(see figure on previous page)

Fig. 6 Interaction of ASF1B and CDK9. a Western blots were performed to confirm the IP efficiency. b SDS-PAGE was conducted to analyze the production of IP. $\mathbf{c}$ Levels of CDK9 mRNA expression in stable ASF1B-shRNA HeLa cells. d. Protein expression of CDK9 in stable ASF1B-shRNA HeLa cells. e. Co-IP was performed to detect CDK9-ASF1B using anti-ASF1B beads and anti-CDK9 beads. $\mathbf{f}$ ASF1B-shRNA-HeLa cells or scrambled cells were treated with $\mathrm{CHX}$ at $20 \mathrm{\mu g} / \mathrm{ml}$ for $0,4,8,12$, and $16 \mathrm{~h}$, and then the CDK9 expression level was determined by western blot. $\mathbf{g}$ ASF1B mediates stabilization through the ubiquitin proteasome pathway. ASF1B-shRNA HeLa cells or control cells were treated with proteasome inhibitor MG132 at $5 \mu \mathrm{M}$ for $4 \mathrm{~h}$, and then the CDK9 expression level was examined by western blot. $\mathbf{h}$ Quantification of the results in (d). Data are mean \pm SEM, $n=3$, and two-tailed unpaired Student's $t$ test was used. ${ }^{* * *} p<0.001$. i Colocalization of ASF1B and CDK9 in the nucleus. Immunofluorescent staining and imaging were used to visualize the colocalization of ASF1B (green fluorescence) and CDK9 (red fluorescence) in stable ASF1B-shRNA HeLa cells and corresponding scrambled cells. $\mathbf{j}$ A schematic model of this work. A schematic diagram showing the signaling pathway of the ASF1B-mediated effect on cervical cancer cell growth via the ASF1B/CDK9 axis.

Table 2 The results of proteome analysis.

\begin{tabular}{|c|c|c|c|c|}
\hline Gene name & Description & Mol. weight $[\mathrm{kDa}]$ & $i B A Q \exp$ & iBAQ igg \\
\hline ASF1B & Histone chaperone ASF1B & 22.433 & 7619100 & 0 \\
\hline $\mathrm{MDH} 2$ & Malate dehydrogenase, mitochondrial & 35.503 & 1319400 & 0 \\
\hline FGFR1OP & FGFR1 oncogene partner & 38.099 & 1285500 & 0 \\
\hline PPP2R1A & Serine/threonine-protein phosphatase $2 \mathrm{~A} 65 \mathrm{kDa}$ regulatory subunit $\mathrm{A}$ alpha isoform & 65.308 & 216090 & 0 \\
\hline PRRC2A & Isoform 2 of Protein PRRC2A & 227.84 & 164310 & 0 \\
\hline RPS17 & $40 \mathrm{~S}$ ribosomal protein $\mathrm{S} 17$ & 15.55 & 1160000 & 0 \\
\hline FOXF1 & Fork head box protein F1 & 40.122 & 836220 & 0 \\
\hline $\mathrm{DH} \times 29$ & ATP-dependent RNA helicase DHX29 & 155.29 & 46364 & 0 \\
\hline SSSCA1 & Sjoegren syndrome/scleroderma autoantigen 1 & 21.474 & 1948100 & 0 \\
\hline ENO1 & Alpha-enolase & 47.168 & 323990 & 0 \\
\hline PCM1 & Pericentriolar material 1 protein & 210.13 & 52791 & 0 \\
\hline PRDX5 & Isoform Cytoplasmic peroxisomal of Peroxiredoxin-5, mitochondrial & 17.031 & 724200 & 0 \\
\hline RAVER1 & Ribonucleoprotein PTB-binding 1 & 77.843 & 101720 & 0 \\
\hline FMR1 & Isoform 4 of Synaptic functional regulator FMR1 & 68.454 & 256320 & 0 \\
\hline YBX3 & Isoform 2 of Y-box-binding protein 3 & 31.947 & 989920 & 0 \\
\hline HNRNPC & Heterogeneous nuclear ribonucleoproteins C1/C2 & 25.256 & 711470 & 0 \\
\hline ENO3 & Beta-enolase (Fragment) & 30.402 & 152620 & 0 \\
\hline LDHB & L-lactate dehydrogenase (Fragment) & 25.218 & 100620 & 0 \\
\hline CDK9 & Cyclin-dependent kinase 9 & 42.777 & 129950 & 0 \\
\hline CPS1 & Isoform 2 of Carbamoyl-phosphate synthase [ammonia], mitochondrial & 116.04 & 15703 & 0 \\
\hline DHX36 & ATP-dependent RNA helicase DHX36 (Fragment) & 91.43 & 20909 & 0 \\
\hline EEF1G & Elongation factor 1-gamma & 50.118 & 76861 & 0 \\
\hline GPI & Glucose-6-phosphate isomerase (Fragment) & 64.824 & 473600 & 0 \\
\hline IQSEC1 & IQ motif and SEC7 domain-containing protein 1 & 91.997 & 52195 & 0 \\
\hline
\end{tabular}

\section{Discussion}

Although some biomarkers, such as SSC-Ag, CA-125, CEA, and cytokeratin, have been reported as markers of cervical cancer ${ }^{44}$, the lack of progress in early diagnosis and treatment reveals the urgent need for increased efforts in cervical cancer research. Previous studies evaluating the effect of ASF1B on cancers revealed that ASF1B functions as an oncogene to promote tumor growth in breast cancers, cell renal cell carcinoma, prostate cancers ${ }^{24,34,35}$. These studies indicated that the high level of ASF1B was correlated with increased rates of cancer progression and metastasis occurrence. However, 
very little was found in the literature describing ASF1B as a pivotal oncogenic gene modulating cervical cancer growth.

In the present study, we first evaluated ASF1B mRNA levels in cervical cancer tumor and para-carcinoma tissues and found that aberrantly high expression of ASF1B occurs in cervical tumors, which was confirmed by qPCR and immunohistochemical analysis (Fig. 1a, b). Subsequently, we induced ASF1B silencing and overexpression to investigate its function in cervical cancer progression. We demonstrated that disruption of ASF1B not only suppressed cervical cancer cell growth in vitro but also slowed cervical cancer cell-derived tumor growth in vivo. These works indicated that ASF1B might promote cervical cancer progression.

Using gain- and loss-of-function strategies, we primarily investigated biological behavior changes, such as proliferation, migration, and cell cycle progression in vitro. We detected that knockdown of ASF1B significantly suppressed cervical cancer cell proliferation and clonogenic survival (Fig. S1a, S1c). We also found that ASF1B knockdown effectively inhibited cervical cancer cell migration by wound healing and transwell assays (Fig. S2a, S2c). In contrast, overexpression of ASF1B promoted proliferation (Fig. S1g) and migration (Fig. S2e, S2g). Importantly, in vivo studies indicate that knockdown of ASF1B in cervical cancer cells obviously slowed tumor growth in the recipient mice (Fig. 3a, b). The study of xenograft tumors in vivo with AAV-shRNA-ASF1B administration further supported that ASF1B silencing leads to the inhibition of tumor growth in cervical cancer cells (Fig. 3f, g). These results suggest that ASF1B is crucial for maintaining cervical cancer cell tumorigenic activity in vitro and promoting tumor growth in vivo.

To elucidate the molecular mechanism of ASF1B knockdown-mediated suppression of cervical cancer cells, protein-protein interaction studies were performed by IP and LC-MS to screen protein groups. As shown in Table 2, we listed approximately 23 proteins involved in the gene regulatory networks of ASF1B. To our surprise, we found that CDK9 could bind to ASF1B. CDK9 belongs to the cyclin-dependent kinase family and is a transcriptional activator recruited to promote RNAPII promoterproximal pause release ${ }^{40}$. Recently, some studies disclosed that CDK9 plays a pivotal role in breast cancer ${ }^{45}$, acute myeloid leukemia ${ }^{46}$, prostate cancer ${ }^{47}$, ovarian cancer ${ }^{48}$, and osteosarcoma ${ }^{42}$. Yamamoto et al. reported that FIT039, CDK9 inhibitor, could inhibit replication of HSV-1, HSV-2, human adenovirus, and human cytomegalovirus in cultured cells ${ }^{49}$. Xu et al. investigated that CDK9 levels are highly correlated with the FIGO stage, pathological grade, deep-stromal invasion, tumor size, and lymph nodes metastasis and knockdown of CDK9 inhibits cervical cancer cell proliferation in vitro, as well as tumorigenesis in vivo ${ }^{36}$. CDK9 was involved in cancer progression through the BRD4-dependent recruitment of $\mathrm{p}$-TEFb for the transcription of the MYC gene, which is a proto-oncogene controlling cell growth and cell cycle progression $^{50}$. In addition, some papers have reported that CDK9 is also involved in the apoptosis pathway through apoptotic or anti-apoptotic proteins, such as cleaved caspase $3^{51}, \mathrm{Bcl}^{52}$, and $\mathrm{Bax}^{53}$. Interestingly, our data also showed that ASF1B suppression caused G2/S stage cell cycle arrest (Figs. 4a, b), and more cells were apoptotic though effects on Bcl2, Bax, and caspase-3 (Fig. 5c). As mentioned in the literature review, the Bcl2/ Bax/caspase3 pathway is an important apoptosis pathway $^{54}$, and our results confirmed that ASF1B disruption mediated by shRNA caused cervical cancer cell apoptosis in this way. Subsequently, we further confirmed that ASF1B and CDK9 are the factors of a nuclear complex to promote cervical cancer progression by co-IP and colocalization of ASF1B and CDK9 in the nucleus (Fig. 6e, i).

Post-translation modifications of proteins, including phosphorylation, acetylation and ubiquitination, play a vital role in controlling numerous biologicals, such as the cell cycle and energy metabolism, among others ${ }^{55}$. Normally, protein ubiquitination acts as a targeting signal to induce protein degradation, in part through the regulation of protein-protein interactions ${ }^{56}$. Kiernan et al. reported that CDK9 is ubiquitinated and degraded by the proteasome whereas cyclin T1 is stable. CDK9 ubiquitination was modulated by cyclin $\mathrm{T} 1$ and $\mathrm{p} 45^{\mathrm{SKP} 2}$. They proposed a novel mechanism whereby recruitment of SCFSKP2 mediated by cyclin $\mathrm{T} 1$ while ubiquitination occurs exclusively on $\mathrm{CDK} 9^{57}$. To investigate whether stability is involved in the relationship of ASF1B-CDK9, we examined CDK9 protein stabilization with $\mathrm{CHX}$ treatment. The results showed that CDK9 was an unstable protein with a half-life of $12 \mathrm{~h}$ (Fig. 6f). All these results propose a novel mechanism that ASF1B might stabilize CDK9 protein by inhibiting its proteasome-mediated ubiquitination and degradation.

\section{Conclusions}

Our study focuses for the first time on the oncogenic activity of ASF1B required to develop cervical cancer tumorigenesis. Disrupting ASF1B can prominently suppress cervical tumor growth by regulating cell cycle and apoptosis pathways. These findings may offer a potential target for developing an anti-tumor cervical cancer therapeutic strategy.

\section{Acknowledgements}

This work is supported by the grants from the National Natural Science Foundation (No. 81773268). 


\section{Author details}

'Department of Pathogen Biology, Nanjing Medical University, 211166 Nanjing, China. ${ }^{2}$ Key Laboratory of Antibody Technique of National Health Commission of China, Nanjing Medical University, 211166 Nanjing, China. ${ }^{3}$ Laboratory Medicine Center, The Second Affiliated Hospital of Nanjing Medical University, 210000 Nanjing, China. ${ }^{4}$ The Affiliated Obstetrics and Gynecology Hospital of Nanjing Medical University, 210004 Nanjing, China. ${ }^{5}$ Department of Medical Biochemistry and Molecular Biology, School of Medicine, Jinan University, 510632 Guangzhou, China

\section{Ethics approval and consent to participate}

The protocol was approved by the Ethics Committee of Nanjing Medical University (No. 2019923) and all patients signed a written informed consent form before specimen collection. The animal care and experimental protocols were approved by the Animal Care and Use Committee of Nanjing Medical University (IACUC-1705003).

\section{Conflict of interest}

The authors declare that they have no conflict of interest.

\section{Publisher's note}

Springer Nature remains neutral with regard to jurisdictional claims in published maps and institutional affiliations.

Supplementary Information accompanies this paper at (https://doi.org/ 10.1038/s41419-020-02872-5).

Received: 23 January 2020 Revised: 29 July 2020 Accepted: 30 July 2020 Published online: 26 August 2020

\section{References}

1. Gan, L., Chen, Y., Liu, H. \& Ju, W. H. Long non-coding R N A ZEB1-antisense 1 affects cell migration and invasion of cervical cancer by regulating epithelialmesenchymal transition via the p38MAPK signaling pathway. Gynecol. Obstet. Invest. 84, 136-144 (2018).

2. Zhang, J. et al. miRNA-218 regulates the proliferation and apoptosis of cervical cancer cells via targeting Gli3. Exp. Ther. Med. 16, 2433-2441 (2018).

3. $\mathrm{Xu}, \mathrm{X}$. et al. Transferrin receptor-involved HIF-1 signaling pathway in cervical cancer. Cancer Gene Ther. 26, 356-365 (2019).

4. Khazaee-Pool, M., Yargholi, F., Jafari, F. \& Ponnet, K. Exploring Iranian women's perceptions and experiences regarding cervical cancer-preventive behaviors. BMC Women's Health 18, 145 (2018).

5. Rotman, J., Mom, C. H., Jordanova, E. S., de Gruijl, T. D. \& Kenter, G. G. 'DURVIT' a phase-I trial of single low-dose durvalumab (Medi4736) IntraTumourally injected in cervical cancer: safety, toxicity and effect on the primary tumourand lymph node microenvironment. BMC Cancer 18, 888 (2018).

6. Zhang, Y. et al. Upregulation of microRNA-148a inhibits proliferation, invasion and migration while promoting apoptosis of cervical cancer cells by downregulating RRS1. Biosci. Rep. 39, BSR20181815 (2019).

7. Bisi-Onyemaechi, A. I., Chikani, U. N. \& Nduagubam, O. Reducing incidence of cervical cancer: knowledge and attitudes of caregivers in Nigerian city to human papilloma virus vaccination. Infect. Agent Cancer 13, 29 (2018).

8. Ruan, G. et al. Cervical cancer screening using the Cervista high-risk human papillomavirus test: opportunistic screening of a hospital-based population in Fujian province, China. Cancer Manag. Res. 10, 3227-3235 (2018).

9. Melnikow, J. et al. Screening for cervical cancer with high-risk human papillomavirus testing: updated evidence report and systematic review for the US preventive services task force. JAMA 320, 687-705 (2018).

10. Zhen, S., Lu, J., Liu, Y. H., Chen, W. \& Li, X. Synergistic antitumor effect on cervical cancer by rational combination of PD1 blockade and CRISPR-Cas9mediated HPV knockout. Cancer Gene Ther. 27, 168-178 (2020).

11. Yang, S., Liu, L., Xu, D. \& Li, X. The relationship of the TLR9 and TLR2 genetic polymorphisms with cervical cancer risk: a meta-analysis of case-control studies. Pathol. Oncol. Res. 26, 307-315 (2018).

12. Luan, X. \& Wang, Y. LncRNA XLOC_006390 facilitates cervical cancer tumorigenesis and metastasis as a ceRNA against miR-331-3p and miR-338-3p. J. Gynecol. Oncol. 29, e95 (2018).
13. Liu, W. B., Wu, J. F., Du, Y. \& Cao, G. W. Cancer evolution-development: experience of hepatitis B virus-induced hepatocarcinogenesis. Curr. Oncol. 23 e49-e56 (2016)

14. Gatenby, R. A. \& Brown, J. Mutations, evolution and the central role of a selfdefined fitness function in the initiation and progression of cancer. Biochim. Biophys. Acta Rev. Cancer 1867, 162-166 (2017).

15. Abascal, F. et al. Subfunctionalization via adaptive evolution influenced by genomic context: the case of histone chaperones ASF1a and ASF1b. Mol. Biol. Evol. 30, 1853-1866 (2013).

16. Hattori, N. \& Ushijima, T. Compendium of aberrant DNA methylation and histone modifications in cancer. Biochem. Biophys. Res. Commun. 455, 3-9 (2014).

17. Meng, C. F., Zhu, X. J., Peng, G. \& Dai, D. Q. Promoter histone H3 lysine 9 dimethylation is associated with DNA methylation and aberrant expression of p16 in gastric cancer cells. Oncol. Rep. 22, 1221-1227 (2009).

18. Gurard-Levin, Z. A., Quivy, J. P. \& Almouzni, G. Histone chaperones: assisting histone traffic and nucleosome dynamics. Annu. Rev. Biochem. 83, 487-517 (2014).

19. Gurard-Levin, Z. A. \& Almouzni, G. Histone modifications and a choice of variant: a language that helps the genome express itself. F1000Prime Rep. 6, 76 (2014).

20. Avvakumov, N., Nourani, A. \& Cote, J. Histone chaperones: modulators of chromatin marks. Mol. Cell 41, 502-514 (2011)

21. Peng, H., Nogueira, M. L., Vogel, J. L. \& Kristie, T. M. Transcriptional coactivator HCF-1 couples the histone chaperone Asf1b to HSV-1 DNA replication components. Proc. Natl Acad. Sci. USA 107, 2461-2466 (2010).

22. Messiaen, S. et al. Loss of the histone chaperone ASF1B reduces female reproductive capacity in mice. Reproduction 151, 477-489 (2016).

23. Paul, P. K. et al. Histone chaperone ASF1B promotes human beta-cell proliferation via recruitment of histone H3.3. Cell Cycle 15, 3191-3202 (2016).

24. Corpet, A. et al. Asf1b, the necessary Asf1 isoform for proliferation, is predictive of outcome in breast cancer. EMBO J. 30, 480-493 (2011).

25. Challagundla, M. et al. AAV-mediated expression of BAG1 and ROCK2-shRNA promote neuronal survival and axonal sprouting in a rat model of rubrospinal tract injury. J. Neurochem. 134, 261-275 (2015).

26. Gui, Z. et al. Oridonin inhibition and miR200b3p/ZEB1 axis in human pancreatic cancer. Int. J. Oncol. 50, 111-120 (2017).

27. Sato, N. et al. Eradication of cervical cancer in vivo by an AAV vector that encodes shRNA targeting human papillomavirus type 16 E6/E7. Int. J. Oncol 52, 687-E6/E696 (2018).

28. Sun, A. J. et al. Adeno-associated virus-delivered short hairpin-structured RNA for androgen receptor gene silencing induces tumor eradication of prostate cancer xenografts in nude mice: a preclinical study. Int. J. Cancer 126, 764-774 (2010).

29. Li, G., Xu, Y., Guan, D., Liu, Z. \& Liu, D. X. HSP70 protein promotes survival of C6 and U87 glioma cells by inhibition of ATF5 degradation. J. Biol. Chem. 286, 20251-20259 (2011).

30. Han, Y. et al. Hepatocyte growth factor increases the invasive potential of PC-3 human prostate cancer cells via an ERK/MAPK and Zeb-1 signaling pathway. Oncol. Lett. 11, 753-759 (2016).

31. Liu, $\mathrm{X}$. et al. Disruption of oncogenic liver-intestine cadherin $(\mathrm{CDH} 17)$ drives apoptotic pancreatic cancer death. Cancer Lett. 454, 204-214 (2019).

32. Yun, D. et al. Shuttling SLC2A4RG is regulated by 14-3-3theta to modulate cell survival via caspase-3 and caspase-6 in human glioma. EBioMedicine 40, 163-175 (2019).

33. Harshman, S. W. et al. Histone $\mathrm{H} 1$ phosphorylation in breast cancer. J. Proteome Res. 13, 2453-2467 (2014)

34. Jiangqiao, Z . et al. Anti-silencing function 1B histone chaperone promotes cell proliferation and migration via activation of the AKT pathway in clear cell renal cell carcinoma. Biochem. Biophys. Res. Commun. 511, 165-172 (2019).

35. Han, G. et al. Knockdown of anti-silencing function 1B histone chaperone induces cell apoptosis via repressing PI3K/Akt pathway in prostate cancer. Int J. Oncol. 53, 2056-2066 (2018)

36. $\mathrm{Xu}$, J. et al. Cyclin-dependent kinase 9 promotes cervical cancer development via AKT2/p53 pathway. IUBMB Life 71, 347-356 (2019).

37. Li, Q. et al. Effects of ambient fine particles PM2.5 on human HaCaT cells. Int. J. Environ. Res. Public Health 14, 72 (2017).

38. Yang, C. et al. Ki67 targeted strategies for cancer therapy. Clin. Transl. Oncol. 20 570-575 (2018) 
39. Lee, S. H. et al. Relationships between lymph node metastasis and expression of CD31, D2-40, and vascular endothelial growth factors A and C in papillary thyroid cancer. Clin. Exp. Otorhinolaryngol. 5, 150-155 (2012).

40. Zhang, $\mathrm{H}$. et al. Targeting CDK9 reactivates epigenetically silenced genes in cancer. Cell 175, 1244-1258 (2018). e1226.

41. Polier, G. et al. Wogonin and related natural flavones are inhibitors of CDK9 that induce apoptosis in cancer cells by transcriptional suppression of $\mathrm{MCl}-1$. Cell Death Dis. 2, e182 (2011)

42. Ma, H., Seebacher, N. A., Hornicek, F. J. \& Duan, Z. Cyclin-dependent kinase 9 (CDK9) is a novel prognostic marker and therapeutic target in osteosarcoma. EBioMedicine 39, 182-193 (2019).

43. Chawsheen, H. A. et al. The redox regulator sulfiredoxin forms a complex with thioredoxin domain-containing 5 protein in response to ER stress in lung cancer cells. J. Biol. Chem. (2019).

44. Dasari, S., Wudayagiri, R. \& Valluru, L. Cervical cancer: biomarkers for diagnosis and treatment. Clin. Chim. Acta 445, 7-11 (2015).

45. Schlafstein, A. J. et al. CDK9 expression shows role as a potential prognostic biomarker in breast cancer patients who fail to achieve pathologic complete response after neoadjuvant chemotherapy. Int J. Breast Cancer 2018, 6945129 (2018).

46. Zhang, X. \& Tao, W. Long noncoding RNA LINC00152 Facilitates the leukemogenesis of acute myeloid leukemia by promoting CDK9 through miR-193a. DNA Cell Biol. 38, 236-242 (2019).

47. Rahaman, M. H. et al. Targeting CDK9: a promising therapeutic opportunity in prostate cancer. Endocr. Relat. Cancer 23, T211-T226 (2016).
48. Wang, J., Dean, D. C., Hornicek, F. J., Shi, H. \& Duan, Z. Cyclin-dependent kinase 9 (CDK9) is a novel prognostic marker and therapeutic target in ovarian cancer. FASEB J. https://doi.org/10.1096/fj.201801789RR (2019).

49. Yamamoto, M. et al. CDK9 inhibitor FIT-039 prevents replication of multiple DNA viruses. J. Clin. Invest 124, 3479-3488 (2014).

50. Franco, L. C., Morales, F., Boffo, S. \& Giordano, A. CDK9: A key player in cancer and other diseases. J. Cell Biochem 119, 1273-1284 (2018).

51. Olson, C. M. et al. Pharmacological perturbation of CDK9 using selective CDK9 inhibition or degradation. Nat. Chem. Biol. 14, 163-170 (2018).

52. Dey, J. et al. Voruciclib, a clinical stage oral CDK9 inhibitor, represses MCL-1 and sensitizes high-risk diffuse large B-cell lymphoma to BCL2 inhibition. Sci. Rep. 7, 18007 (2017).

53. Shiozaki, Y. et al. The CDK9-cyclin T1 complex mediates saturated fatty acidinduced vascular calcification by inducing expression of the transcription factor CHOP. J. Biol. Chem. 293, 17008-17020 (2018).

54. Chen, Y. et al. Porphyromonas gingivalis induced inflammatory responses and promoted apoptosis in lung epithelial cells infected with H1N1 via the Bcl2/ Bax/Caspase3 signaling pathway. Mol. Med Rep. 18, 97-104 (2018).

55. Peng, Y. et al. Characterization of Osterix protein stability and physiological role in osteoblast differentiation. PLoS ONE 8, e56451 (2013).

56. Burton, J. C. \& Grimsey, N. J. Ubiquitination as a key regulator of endosomal signaling by GPCRs. Front Cell Dev. Biol. 7, 43 (2019).

57. Kiernan, R. E. et al. Interaction between cyclin T1 and SCF(SKP2) targets CDK9 for ubiquitination and degradation by the proteasome. Mol. Cell Biol. 21, 7956-7970 (2001) 